\title{
Epidemic Dynamics for the Two-stage Model on Scale-free Networks
}

\author{
Maoxing Liu $^{\mathrm{a}}$ \\ Department of Mathematics of North University of China, Taiyuan, P. R. China \\ Email: liumaoxing@126.com \\ Yunli Zhang \\ Department of Mathematics of North University of China, Taiyuan, P. R. China \\ Email: zhangyunli5365@sina.com \\ Wei Han \\ Department of Mathematics of North University of China, Taiyuan, P. R. China \\ Email: qd_hanweiweil@126.com
}

\begin{abstract}
In this paper, we will study a two-stage model on complex networks. The dynamic behaviors of the model on a heterogeneous scale-free (SF) network are considered, where the absence of the threshold on the SF network is demonstrated, and the stability of the disease-free equilibrium is obtained. Four immunization strategies, proportional immunization, targeted immunization, acquaintance immunization and active immunization are applied in this model. We show that both targeted and acquaintance immunization strategies compare favorably to a proportional scheme in terms of effectiveness. For active immunization, the threshold is easier to apply practically.
\end{abstract}

Index Terms-Complex network; Epidemic; Two-stage model; Threshold

\section{INTRODUCTION}

Mathematical models have been applied to the study of infectious diseases since more than a century ago. The last four decades have witnessed a burst of interest in quantitatively understanding the transmission dynamics of a large number of diseases [1]. One of the key aims of epidemiological mathematical models, and certainly the most relevant in terms of policy making, is the assessment of the effectiveness of control strategies to curb disease spreading. For many infectious diseases, the most widespread prevention measure is mass vaccination. However, if for a given disease vaccines are not known, or vaccination is not effective, other control measures have to be adopted.

Compartmental model is often used to describe the spread of infectious diseases, such as HIV, rabies, SARS, TB. In 1927, Kermack and McKendrick suggested an SIR model, which well indicated the number of infected population during the plague [2]. The contact process was introduced by Harris [3]. In a contact process, individuals

* Project supported by the National Natural Science Foundation of China(No. 10901145)and the Top Young Academic Leaders of Higher Learning Institutions of Shanxi.

a. Corresponding authors in the population have two life stages, young and adult, only adults can give birth and each new offspring is young. Transition from young to adult occurs at constant rate, and individuals die at rates that depend on their life stage. Krone[6] gives a very nice analysis of this process by the construction of a multi-type dual process. Models of this class have proven particularly useful for the study of Chlamydia and gonorrhea [4], [5].

We consider a population formed by $\mathrm{N}$ agents, situated at the nodes of a network. Individuals are modeled as nodes, and possible contacts between individuals are linked by edges. It has been shown that there is an epidemic threshold on the homogeneous Watts-Strogatz (WS) small-world network model, while the epidemic model on SF networks has no epidemic threshold; infections can be proliferated, whatever small infection rate they have. This result disproves the threshold theory in epidemiology. Recently, there are some related research works on complex networks, for example, with infective medium [14], even with two species or pathogens spreading [15], [16].

These studies have extended and detailed the epidemic model on complex networks. It has shown that networks of two-stage contact process are scale-free [6], [7], [8], and [17] i.e. the distribution of the number of contacts over all individuals in the network has a long upper tail which exhibits power-law behavior. To study such a spreading characteristic, this paper extends the standard model to qualitatively understand and describe the mechanism of epidemics spreading based on biological means. It is shown that the main features and theoretical results obtained here are different from those in general SIS models [7], [8], [9].

The state-transition rules of the two-stage contact process have been put forward in Ref. [17]. It differs from the SIRS model in that the transition rate from 0 to 1 is proportional to the density of the neighbors with state 2 (not 1 ) of a state- 0 vertex. This model corresponds to dynamics with two life stages. The states can be interpreted as 0 : vacant, 1: occupied by young individuals, 
and 2: occupied by adults. Only adults are reproductive and generate offspring in neighboring vacant sites at a birth rate equal to $\lambda$. In other words, a birth event occurs at a vacant site at a rate proportional to $\lambda$ and the number of neighboring adults. Younger (state 1) spend random time of mean $\gamma$ before becoming adult (state 2). They are also subject to random death events at a rate of $\delta$. Adults die at a rate of 1 , which gives normalization of the entire model. Alternatively, we can interpret the three states as 0: vacant, 1: partially occupied, and 2: fully occupied colonies. Then, only fully occupied colonies are potent enough to colonize vacant lands. Based on these state-transition rules, we will establish and consider this model on scale-free networks.

The organization of this paper is as follows: Section 2 derives the mean-field epidemic spreading model of SF network, especially Barabasi-Albert (BA) model with connectivity $p(k): k^{-3}$ is obtained, and the existence of the steady-state solution is analyzed. Section 3 discusses the stability of the disease-free equilibrium. In Section 4 we consider several models of immunization. Finally, a brief discussion is given in Section 5.

\section{THE MODEL}

To derive mean field dynamics for populations with heterogeneous contact rates, let us denote by $p_{k}$ the probability that a vertex has degree $k$. Obviously, $\sum_{k=1}^{N} p_{k}=1$.

In this paper, there are two types of nodes in a network, one is composed of $\mathrm{N}$ first-stage individuals and the other is composed of $\mathrm{N}$ second-stage individuals. All nodes can exist only in one of the two discrete states, healthy or infected. And the disease transmission is described as in the paper [7], [8]: At each time step, each susceptible (healthy) node is infected with rate $\boldsymbol{V}$ if it is connected to one or more infected nodes. At the same time, infected nodes are cured and become again susceptible with rate $\tau$ defining an effective spreading rate $\lambda=v / \tau$. Without lack of generality, we can set $\tau=1$. All nodes in this network run stochastically through the healthy-infected-healthy cycles. So in this paper, we are interested in studying the following dynamical model

$$
\begin{aligned}
& \beta_{1, \mathrm{k}}=\lambda\left(1-\rho_{1, \mathrm{k}}-\rho_{2, \mathrm{k}}\right) \mathrm{k} \theta-(\delta+\gamma) \rho_{1, \mathrm{k}}, \\
& \beta_{2, \mathrm{k}}=\gamma \rho_{1, \mathrm{k}}-\rho_{2, \mathrm{k}} .
\end{aligned}
$$

We note that the effective birth rate (the first term in the first equation (1a) is proportional to $k \theta$, which is the average number of state- 2 vertices in the neighborhood of a degree- $k$ vertex. When we choose an arbitrary edge, the probability that a specific vertex is connected to this edge is proportional to its degree $\mathrm{k}$ [7], [8], [10], [11],
[13], where $\rho_{1, \mathrm{k}}\left(\rho_{2, \mathrm{k}}\right)$ denotes the density of infected first-stage and second-stage individuals with connectivity $\mathrm{k}$ in the networks, the edge-conditioned probability $0 \leq \theta \leq 1$ describes a link pointing to an infected individual [7], [8], which satisfies:

$$
\theta(\mathrm{t})=\frac{\sum_{\mathrm{k}} \mathrm{kp}_{\mathrm{k}} \rho_{2, \mathrm{k}}(\mathrm{t})}{\langle\mathrm{k}\rangle} .
$$

where $\langle k\rangle=\sum_{k} k p_{k}, p_{k}$ is the connectivity distribution of the vertex individuals.

In the steady state

$$
\boldsymbol{\alpha}_{\mathrm{t}, \mathrm{k}}=0, \boldsymbol{\alpha}_{2, \mathrm{k}}=0 \text {, }
$$

one has

$$
\begin{gathered}
\lambda\left(1-\rho_{1, \mathrm{k}}-\rho_{2, \mathrm{k}}\right) \mathrm{k} \theta-(\delta+\gamma) \rho_{1, \mathrm{k}}=0, \\
\gamma \rho_{1, \mathrm{k}}-\rho_{2, \mathrm{k}}=0 .
\end{gathered}
$$

It is easy to see that model has a disease-free equilibrium: $\mathrm{E}_{0}=(0, \mathrm{~L}, 0)$. As far as the possibility of spreading is concerned, the following lemma holds:

Theorem 2.1. If and only if

$$
\lambda>\frac{(\delta+\gamma)\langle\mathrm{k}\rangle}{\gamma\left\langle\mathrm{k}^{2}\right\rangle}
$$

then there exists a unique endemic equilibrium $\mathrm{E}^{*}=\left(\mathrm{X}_{1}^{*}, \mathrm{~L}, \mathrm{X}_{\mathrm{N}}^{*}, \mathrm{y}_{1}^{*}, \mathrm{~L}, \mathrm{y}_{\mathrm{N}}^{*}\right)$.

proof: From (4), we can get

$$
\begin{aligned}
& \rho_{1, \mathrm{k}}=\frac{\lambda \mathrm{k} \theta}{\lambda(1+\gamma) \mathrm{k} \theta+\delta+\gamma}, \\
& \rho_{2, \mathrm{k}}=\frac{\gamma \lambda \mathrm{k} \theta}{\lambda(1+\gamma) \mathrm{k} \theta+\delta+\gamma} .
\end{aligned}
$$

And substituting them into (2), we get

$$
\theta=\frac{1}{\langle\mathrm{k}\rangle} \sum_{\mathrm{k}} \frac{\lambda \mathrm{k}^{2} \theta \mathrm{p}_{\mathrm{k}}}{\lambda(1+\gamma) \mathrm{k} \theta+\delta+\gamma} \equiv \mathrm{f}(\theta) \text {. }
$$

For Eq.(6) is satisfied when $\theta=0$, corresponding to the disease-free state. When $0<\theta<1$ state 2 survives, Eq.(6) implies $\rho_{1, \mathrm{k}}>0, \rho_{2, \mathrm{k}}>0$ in this situation. Accordingly, $\theta>0$ is equivalent to the $\{0,1,2\}$ phase. It is easy to verify that $f(\theta)$ is a convex and increase function, and $\left.f(\theta)\right|_{\theta=1}<1$, so if there is another solution $0<\theta<1$, it must satisfy $\left.\frac{\mathrm{df}(\theta)}{\mathrm{d} \theta}\right|_{\theta=0}>1$, that is

$$
\lambda>\frac{(\delta+\gamma)\langle\mathrm{k}\rangle}{\gamma\left\langle\mathrm{k}^{2}\right\rangle}
$$

where $\left\langle k^{2}\right\rangle=\sum_{k} k^{2} p_{k}$. Through the unique endemic 
equilibrium $E^{*}$ is determined. This agrees with the results for the percolation, the contact process, and the SIR model.

Remark 2.1.

(1) Let

$$
\mathrm{R}=\frac{\lambda \gamma\left\langle\mathrm{k}^{2}\right\rangle}{(\delta+\gamma)\langle\mathrm{k}\rangle}
$$

In mathematical biology, $\mathrm{R}$ is called the basic productive number, which determines whether the disease is transmittable or not.

(2) If we consider $\mathrm{p}_{\mathrm{k}}=\mathrm{Ck}^{-\mathrm{v}} \boldsymbol{\delta}_{\mathrm{k}, \mathrm{k}_{\mathrm{c}}}\left(\mathrm{k}_{\mathrm{c}} \geq 2\right)$. The network is homogenous, $\langle k\rangle=k_{c}$, so

$$
\mathrm{R}=\frac{\lambda \gamma \mathrm{k}_{\mathrm{c}}}{\delta+\gamma}
$$

When $R<1$, the disease-free equilibrium is local asymptotically stable; while $R>1$, there is an endemic equilibrium. Moreover, $\mathrm{R}$ is an increasing function of $\lambda$ and $k_{c}$. In order to make $R<1$, we need decrease $\lambda$ and $k_{c}$.

(3) By using a continuous $\mathrm{k}$ approximation that allows a practical substitution of series with integrals [18], the full connectivity distribution can be obtained as $p_{k}=2 m^{2} / k^{3}$ where $m$ is the minimum number of connections at each individual node, and

$$
\langle k\rangle=\int_{m}^{\infty} k p_{k} d k=2 m \text {. }
$$

Furthermore, here

$$
\left\langle k^{2}\right\rangle ; 2 m^{2} \ln \left(k_{c} / m\right) .
$$

Substituting them into yields

$$
\mathrm{R}=\frac{\lambda \gamma \mathrm{m} \ln \left(\mathrm{k}_{\mathrm{c}} / \mathrm{m}\right)}{\delta+\gamma}
$$

If we consider the maximum connectivity $k_{c}$ to $N$ by $\mathrm{k}_{\mathrm{c}} \mathrm{m} \sqrt{\mathrm{N}}$, so

$$
\mathrm{R}=\frac{\lambda \gamma \mathrm{m} \ln (\mathrm{N})}{2(\delta+\gamma)}
$$

\section{THE STABILITY OF THE DISEASE-FREE}

\section{EQUILIBRIUM (DFE)}

In this section we consider the stability of the diseasefree equilibrium. First, Jacobin matrix at an equilibrium is

and where

$$
J=\left(\begin{array}{ll}
A & B \\
C & D
\end{array}\right),
$$

$$
\mathrm{A}=\left(\begin{array}{ccc}
-\lambda \theta-(\delta+\gamma) & \mathrm{L} & 0 \\
\mathrm{M} & \mathrm{O} & \mathrm{M} \\
0 & \mathrm{~L} & -\mathrm{N} \lambda \theta-(\delta+\gamma)
\end{array}\right),
$$

$B=B_{1}+B_{2}$, where

$$
\mathrm{B}_{1}=\left(\begin{array}{ccc}
-\lambda \theta & \mathrm{L} & 0 \\
\mathrm{M} & \mathrm{O} & \mathrm{M} \\
0 & \mathrm{~L} & -\mathrm{N} \lambda \theta
\end{array}\right)
$$

$$
B_{2}=\frac{\lambda}{\langle k\rangle}\left(\begin{array}{ccc}
p_{1} h_{1} & L & N p_{N} h_{N} \\
M & O & M \\
N p_{1} h_{N} & L & N^{2} p_{N} h_{N}
\end{array}\right),
$$

where $h_{\mathrm{i}}=1-\rho_{1, \mathrm{i}}-\rho_{2, \mathrm{i}},(\mathrm{i}=1, \mathrm{~L}, \mathrm{~N})$.

$$
\begin{aligned}
& C=\gamma\left(\begin{array}{ccc}
1 & L & 0 \\
M & O & M \\
0 & L & 1
\end{array}\right), \\
& D=\left(\begin{array}{ccc}
-1 & L & 0 \\
M & O & M \\
0 & L & -1
\end{array}\right) .
\end{aligned}
$$

So we can easily get the Jacobin matrix at disease-free equilibrium is

where

$$
J^{\prime}=\left(\begin{array}{ll}
A^{\prime} & B^{\prime} \\
C^{\prime} & D^{\prime}
\end{array}\right),
$$

$$
\begin{gathered}
\mathrm{A}^{\prime}=\left(\begin{array}{ccc}
-(\delta+\gamma) & \mathrm{L} & 0 \\
\mathrm{M} & \mathrm{O} & \mathrm{M} \\
0 & \mathrm{~L} & -(\delta+\gamma)
\end{array}\right), \\
\mathrm{B}^{\prime}=\frac{\lambda}{\langle\mathrm{k}\rangle}\left(\begin{array}{ccc}
\mathrm{p}_{1} & \mathrm{~L} & \mathrm{~N} \mathrm{p}_{\mathrm{N}} \\
\mathrm{M} & \mathrm{O} & \mathrm{M} \\
\mathrm{N} \mathrm{p}_{1} & \mathrm{~L} & \mathrm{~N}^{2} \mathrm{p}_{\mathrm{N}}
\end{array}\right),
\end{gathered}
$$

$$
C^{\prime}=\gamma\left(\begin{array}{lll}
1 & L & 0 \\
M & O & M \\
0 & L & 1
\end{array}\right)
$$




$$
D^{\prime}=\left(\begin{array}{ccc}
-1 & L & 0 \\
M & O & M \\
0 & L & -1
\end{array}\right) \text {. }
$$

The matrix $J^{\prime}$ has $2 \mathrm{~N}-1$ eigenvalues equal to $i 1$ : $\mu_{\mathrm{i}}=-1(\mathrm{i}=1, \mathrm{~L} 2 \mathrm{~N}-1)$, the $2 \mathrm{~N}$ th is

$$
\mu_{2 \mathrm{~N}}=-1+\frac{\lambda \gamma\left\langle\mathrm{k}^{2}\right\rangle}{(\delta+\gamma)\langle\mathrm{k}\rangle} \text {. }
$$

Having established these premises, we may immediately demonstrate the following theorem:

Theorem 3.1. If $R \leq 1$ then the disease-free equilibrium of (1) is asymptotically stable in the set $[0,1]^{2 \mathrm{~N}}$, otherwise there exists a unique endemic equilibrium $E^{*}=\left(x_{1}^{*}, L, x_{N}^{*}, y_{1}^{*}, L, y_{N}^{*}\right)$.

\section{Remark 3.1.}

The biological consequence of this result is that if the epidemic threshold $R$ is not exceeded the disease will disappear. On the contrary if $R>1$, then there is an endemic solution which is reached independently of the initial state of the disease. In other words, however complex the system may be and whatever the initial state of the first-stage and the second-stage individuals are, whether the disease will eradicate or not only depend on the basic reproduction number.

\section{IMMUNIZATION STRATEGIES}

Vaccination is very useful in controlling vaccine preventable diseases. The SIS model is more appropriate than the susceptible-infected-recovered model in the early stage of epidemic outbreaks when the effects of recovery and death can be ignored, and this is the optimal time period for immunization to be applied. In this section we discuss the SIS model with two types of nodes on a scalefree network, and various immunization schemes [9].

\section{A. Proportional immunization}

Denote by $g$ the immunization rate, $0<\mathrm{g}<1$; the presence of proportional immunity will effectively reduce the spreading rate $\lambda$ by a factor $\lambda(1-\mathrm{g})$, then Eq. (1) becomes

$\beta_{1, \mathrm{k}}=\lambda(1-\mathrm{g})\left(1-\rho_{1, \mathrm{k}}-\rho_{2, \mathrm{k}}\right) \mathrm{k} \theta-(\delta+\gamma) \rho_{1, \mathrm{k}}$,

$\delta_{2, \mathrm{k}}=\gamma \rho_{1, \mathrm{k}}-\rho_{2, \mathrm{k}}$.

Let $\delta_{\mathrm{q}, \mathrm{k}}=0, \boldsymbol{\alpha}_{2, \mathrm{k}}=0$, from (11) we have

$$
\rho_{1, \mathrm{k}}=\frac{\lambda \mathrm{k} \theta(1-\mathrm{g})}{\lambda(1+\gamma)(1-\mathrm{g}) \mathrm{k} \theta+\delta+\gamma},
$$

$$
\rho_{2, \mathrm{k}}=\frac{\gamma \lambda \mathrm{k} \theta(1-\mathrm{g})}{\lambda(1+\gamma)(1-\mathrm{g}) \mathrm{k} \theta+\delta+\gamma},
$$

Substitute $\rho_{1, \mathrm{k}}, \rho_{2, \mathrm{k}}$ in (2) by (12), we obtain a selfconsistency equation as follows:

$$
\theta=\frac{1}{\langle\mathrm{k}\rangle} \sum_{\mathrm{k}} \frac{\lambda \mathrm{k}^{2} \theta p_{\mathrm{k}}(1-\mathrm{g})}{\lambda(1+\gamma)(1-\mathrm{g}) \mathrm{k} \theta+\delta+\gamma} \equiv \tilde{\mathrm{f}}(\theta) .
$$

By arguments similar to those in Sec. II, the epidemic threshold $\tilde{\lambda}_{\mathrm{c}}$ is determined by the following inequality:

$$
\left.\frac{\mathrm{d} \tilde{\mathrm{f}}(\theta)}{\mathrm{d} \theta}\right|_{\theta=0}>1
$$

therefore, it can be shown that

$$
\tilde{\lambda}_{\mathrm{c}}=\frac{(\delta+\gamma)\langle\mathrm{k}\rangle}{\gamma(1-\mathrm{g})\left\langle\mathrm{k}^{2}\right\rangle}
$$

that is,

$$
\tilde{\lambda}_{\mathrm{c}}=\frac{1}{1-\mathrm{g}} \lambda_{\mathrm{c}} .
$$

Note that in (14), when $g=0$, i.e., if no immunization were done, then $\tilde{\lambda}_{\mathrm{c}}=\lambda_{\mathrm{c}}$; when $0<\mathrm{g}<1, \tilde{\lambda}_{\mathrm{c}}>\lambda_{\mathrm{c}}$, that is, the immunization scheme is effective; while as $\mathrm{g} \rightarrow 1, \tilde{\lambda}_{\mathrm{c}} \rightarrow+\infty$, that is, in the case of a full immunization, it would be impossible for the epidemic to spread in the network.

\section{B. Targeted immunization}

While proportional immunization schemes are effective, there may be more efficient schemes due to the heterogeneous nature of scale-free networks: they are robust to random attacks, but fragile to selective attacks. Accordingly, we can devise a targeted immunization scheme [9]. We introduce an upper threshold $\kappa$, such that all nodes with connectivity $k>K$ are immunized, i.e., we define the immunization rate $g_{k}$ by

$$
g_{\mathrm{k}}=\left\{\begin{array}{l}
1, \mathrm{k}>\kappa \\
\mathrm{c}, \mathrm{k}=\kappa \\
0, \mathrm{k}<\kappa
\end{array}\right.
$$

where $0<c \leq 1$, and $\sum_{k} g_{k} p_{k}=\bar{g}$, where $\bar{g}$ is the average immunization rate. The epidemic dynamics model is

$$
\begin{aligned}
& \delta_{1, \mathrm{k}}=\lambda\left(1-\mathrm{g}_{\mathrm{k}}\right)\left(1-\rho_{1, \mathrm{k}}-\rho_{2, \mathrm{k}}\right) \mathrm{k} \theta-(\delta+\gamma) \rho_{1, \mathrm{k}}, \\
& \delta_{\mathrm{\alpha}, \mathrm{k}}=\gamma \rho_{1, \mathrm{k}}-\rho_{2, \mathrm{k}} .
\end{aligned}
$$

This lead to

$$
\theta=\frac{1}{\langle k\rangle} \sum_{k} \frac{\lambda k^{2} \theta p_{k}\left(1-g_{k}\right)}{\lambda(1+\gamma)\left(1-g_{k}\right) k \theta+\delta+\gamma} \equiv \hat{f}(\theta) .
$$


Therefore, the epidemic threshold

$$
\hat{\lambda}_{\mathrm{c}}=\frac{(\delta+\gamma)\langle\mathrm{k}\rangle}{\gamma\left(1-\mathrm{g}_{\mathrm{k}}\right)\left\langle\mathrm{k}^{2}\right\rangle}=\frac{(\delta+\gamma)\langle\mathrm{k}\rangle}{\gamma\left\langle\mathrm{k}^{2}\right\rangle-\gamma\left\langle\mathrm{g}_{\mathrm{k}} \mathrm{k}^{2}\right\rangle}
$$

Note that $\left\langle g_{k} k^{2}\right\rangle=\bar{g}\left\langle k^{2}\right\rangle+\sigma^{\prime} \quad$,where $\sigma^{\prime}=\left\langle\left(g_{k}-\bar{g}\right) \times\left[k^{2}-\left\langle k^{2}\right\rangle\right]\right\rangle$ is the covariance of $g_{k}$ and $k^{2}$, There may be $\kappa$ (usually big enough) where $\sigma^{\prime}<0$, but for appropriately small $\kappa, g_{k}-\bar{g}$ and $k^{2}-\left\langle k^{2}\right\rangle$ have the same signs except for some $k$ 's.

Where $g_{k}-\bar{g}$ and / or $k^{2}-\left\langle k^{2}\right\rangle$ is zero; therefore for $\sigma^{\prime}>0$ appropriate $\kappa$. Then

$$
\hat{\lambda}_{c}>\frac{1-g}{1-\bar{g}} \tilde{\lambda}_{c} .
$$

If we set $g=\bar{g}$, then $\hat{\lambda}_{c}>\tilde{\lambda}_{c}(0<g<1)$, which means the targeted immunization scheme is more efficient than the proportional scheme.

\section{Acquaintance immunization}

As discussed in [22], [23], a problem with the targeted immunization scheme is that it requires some global information about the degree of each node. In this section, we use another immunization strategy, acquaintance immunization [12], which calls for the immunization of random acquaintances of random nodes.

Choose a random fraction $\mathrm{p}$ of the $\mathrm{N}$ nodes; the probability that a particular node with $k$ contacts is selected for immunization is $k p(k) /(N\langle k\rangle)[24,25]$. Therefore, in (16) we may take

$$
g_{k}=\frac{k p(k)}{N\langle k\rangle} p N=\frac{p}{\langle k\rangle} k p(k),
$$

so the epidemic threshold for this immunization scheme is

$$
\lambda_{\mathrm{c}}=\frac{(1-\bar{g}) \gamma\left\langle\mathrm{k}^{2}\right\rangle-\gamma \sigma^{\prime}}{\gamma\left\langle\mathrm{k}^{2}\right\rangle-\frac{p}{\langle\mathrm{k}\rangle \zeta(2+\gamma) \mathrm{k}^{1+\gamma}} \cdot\left\langle\mathrm{k}^{2}\right\rangle} \hat{\lambda}_{\mathrm{c}} .
$$

Note that

$$
\begin{aligned}
& (1-\bar{g}) \gamma\left\langle\mathrm{k}^{2}\right\rangle-\gamma \sigma^{\prime} \\
= & (1-\bar{g}) \gamma\left\langle\mathrm{k}^{2}\right\rangle-\gamma\left\langle\left(g_{\mathrm{k}}-\overline{\mathrm{g}}\right) \times\left[\mathrm{k}^{2}-\left\langle\mathrm{k}^{2}\right\rangle\right]\right\rangle \\
> & (1-\bar{g}) \gamma\left\langle\mathrm{k}^{2}\right\rangle-\gamma\left\langle(1-\overline{\mathrm{g}}) \times\left[\mathrm{k}^{2}-\left\langle\mathrm{k}^{2}\right\rangle\right]\right\rangle \\
> & \gamma(1-\overline{\mathrm{g}})\left\langle\mathrm{k}^{2}\right\rangle-\gamma\left\langle(1-\overline{\mathrm{g}}) \mathrm{k}^{2}\right\rangle=0, \\
& \gamma\left\langle\mathrm{k}^{2}\right\rangle-\frac{2 p}{\langle\mathrm{k}\rangle \zeta(2+\gamma) \mathrm{k}^{1+\gamma}} \cdot\left\langle\mathrm{k}^{2}\right\rangle>\gamma\left\langle\mathrm{k}^{2}\right\rangle-\gamma\left\langle\mathrm{k}^{-1-\gamma} \cdot \mathrm{k}^{2}\right\rangle>0 \\
& \text { So }, \lambda_{\mathrm{c}}=\Lambda \hat{\lambda}_{\mathrm{c}}, \text { where } \Lambda \text { is a positive constant. }
\end{aligned}
$$

This means the acquaintance immunization scheme is comparable in effectiveness to the targeted immunization scheme.

\section{Active immunization}

In this section we propose a different immunization scheme: choose an infected node and immunize its neighbors whose degree $\geq K$. That is, the epidemic dynamics model is

$$
\begin{aligned}
& \delta_{1, \mathrm{k}}=\lambda\left(1-\rho_{1, \mathrm{k}}-\rho_{2, \mathrm{k}}\right) \mathrm{k} \theta-(\delta+\gamma) \rho_{1, \mathrm{k}}, \\
& \boldsymbol{\beta}_{2, \mathrm{k}}=\gamma \rho_{1, \mathrm{k}}-\left(1+\bar{g}_{\mathrm{k}^{\prime}}\right) \rho_{2, \mathrm{k}} .
\end{aligned}
$$

where

$$
g_{k}=\sum_{k^{\prime}} \frac{k^{\prime} p\left(k^{\prime}\right)}{\langle k\rangle} g_{k^{\prime}},
$$

and $g_{k^{\prime}}$ is defined in (15).

After the early stage of a disease epidemic, there may be quite a lot of infected individuals; therefore this immunization scheme may be more appropriate. We show this rigorously below.

By letting $\oint_{1, \mathrm{k}}=0, \boldsymbol{\alpha}_{2, \mathrm{k}}=0$, model (18) leads to

$$
\begin{aligned}
\theta & =\sum_{k^{\prime}} \frac{\gamma \lambda k^{\prime 2} p_{k^{\prime}} \theta}{\left[\left(1+\bar{g}_{k^{\prime}}\right) \lambda k \theta+\gamma \lambda k \theta+(\delta+\gamma)\left(1+\bar{g}_{k^{\prime}}\right)\right] \cdot\langle k\rangle} \\
& \equiv \bar{f}(\theta),
\end{aligned}
$$

therefore, the epidemic threshold

$$
\bar{\lambda}_{\mathrm{c}}=\frac{\langle\mathrm{k}\rangle}{\left\langle\mathrm{k}^{2}\right\rangle} \cdot \frac{(\delta+\gamma)\left(1+\bar{g}_{\mathrm{k}^{\prime}}\right)}{\gamma}
$$

We have

$$
\bar{\lambda}_{\mathrm{c}}=\frac{\langle\mathrm{k}\rangle}{\left\langle\mathrm{k}^{2}\right\rangle} \cdot \frac{\delta+\gamma}{\gamma}+\frac{\langle\mathrm{k}\rangle}{\left\langle\mathrm{k}^{2}\right\rangle} \cdot \frac{(\delta+\gamma) \overline{\mathrm{g}}_{\mathrm{k}^{\prime}}}{\gamma} .
$$

Compare (19) with $\lambda_{c}=\frac{(\delta+\gamma)\langle k\rangle}{\gamma\left\langle k^{2}\right\rangle}$, we have

$$
\bar{\lambda}_{\mathrm{c}}=\lambda_{\mathrm{c}}+\bar{g}_{\mathrm{k}^{\prime}} \cdot \lambda_{\mathrm{c}}>\lambda_{\mathrm{c}} \text {. }
$$

That is to say, the immunization scheme we propose here is indeed effective, and the lower $\kappa$, the greater the term $\bar{g}_{k^{\prime}}$ is and the more effective the scheme.

\section{E. A brief summary}

In previous sections we have discussed proportional, targeted, acquaintance, and active immunization schemes, and estimated the thresholds for each scheme. By comparing the thresholds for different immunization schemes, we have concluded that the targeted immunization scheme is more efficient than the proportional scheme; the acquaintance immunization scheme is comparable to the targeted immunization scheme; and the effectiveness of the active immunization scheme is also discussed.

\section{CONCLUSION}


In this paper, a new model for two-stage contact process on complex networks has been proposed and simulated. Different from the classical epidemic model, in the new model infection between two-stage contact processes is taken into account. Moreover, spreading thresholds of the two-stage contact process model are determined, and the stability of the disease-free equilibrium is given, but as to the stability of the endemic equilibrium, which can show the global behavior of the solutions of the model, we have not mentioned in this paper. These and some other related issues will be further studied in the future.

In [22] a probability approach is used to calculate epidemic thresholds for random, targeted, and acquaintance immunization schemes, which are critical probability values and can be used to evaluate the fraction of immunized individuals. While in [9] proportional and targeted immunization schemes are discussed, epidemic thresholds are not considered directly; instead as in [22], the critical fractions of immunized individuals are discussed. Here, we give a direct characterization of epidemic thresholds for more immunization schemes, including the scheme of active immunization, so the thresholds are easier to apply practically.

\section{ACKNOWLEDGMENT}

The authors would like to thank the referee for his/her valuable comments which have led to an improvement of the presentation of this paper.

\section{REFERENCES}

[1] R. M. Anderson, R. M. May, Infectious Diseases of Humans, Oxford University Press, Oxford, UK (1991).

[2] Kermack, W. O. and A. G. McKendrick. 1927. A Contribution to the Mathematical. Theory of Epidemics. Proc. Roy. Soc. A. 115, 700-721.

[3] T. Harris, Contact interactions on a lattice, Ann. Probab., 2 (1974) 969-988.

[4] H. W. Hethcote, J. A. Yorke, Gonorrhoea: transmission dynamics and control in: Lecture Notes in Biomathematics, vol. 56. Springer, New York, 1984.

[5] M. Kretzschmar, Y.T.H.P. Van Duynhoven, A. J. Severijnen, Modeling prevention strategies for gonorrhoea and chlamydia using stochastic network simulations. Am. J. Epidemiol., 144 (1997) 306-317.

[6] S. M. Krone, The two-stage contact process, The Annals of Applied Probability, 9 (2) 1999 331-351.

[7] R. Pastor-Satorras, A. Vespignani, Epidemic spreading in scale-free networks, Phys. Rev. Lett., 86 (2001a) 32003203.

[8] R. Pastor-Satorras, A. Vespignani, Epidemic dynamics and endemic states in complex networks, Phys. Rev. E, 63 (2001b) 066117.

[9] R. Pastor-Satorras, A. Vespignani, Immunization of complex networks,Phys. Rev. E, 65 (2002) 036104.

[10] D. S. Callaway, M. E. J. Newman, S. H. Strogatz, D. J. Watts, Network robustness and fragility: percolation on random graphs, Phys. Rev. Lett., 85 (2000) 5468-5471.

[11] R. Cohen, K. Erez, D. Ben-Avraham, S. Havlin, Resilience of the Internet to random breakdowns, Phys. Rev. Lett., 85 (2000) 4626-4628.
[12] R. Cohen, S. Havlin, D. Ben-Avraham, , Phys. Rev. Lett., 91 (2003)247901.

[13] M. E. J. Newman, Spread of epidemic disease on networks, Phys. Rev.E, 66 (2002) 016128.

[14] H. Shi, Z. Duan, G. Chen, An SIS model with infective medium on complex networks, Physica A 387 (2008) 2133-2144.

[15] Y. Y. Ahn, H. Jeong, N. Masuda, J. D. Noh, Epidemic dynamics of two species of interacting particles on scalefree networks, Phys. Rev. E, 74(2006) 066113.

[16] M. E. J. Newman, Threshold Effects for Two Pathogens Spreading on a Network, Phys. Rev. Lett., 95 (2005) 108701.

[17] N.K. Masuda, N. Konno, Multi-state epidemic processes on complex networks, Journal of Theoretical Biology, 243 (2006) 64-75.

[18] A. -L. Barabasi, R. Albert, Emergence of scaling in random networks, Science 286 (1999) 509-512.

[19] W. P. Guo, X. Li, X. F. Wang, Epidemics and immunization on Euclidean distance preferred small-world networks, Physica A 380 (2007) 684-690.

[20] X. L, X. F. Wang, Controlling the spreading in small-world evolving networks: stability, oscillation, and topology, IEEE Trans. Automat. Control 51 (3) (2006) 534-540.

[21] X. L, X. F. Wang, On the stability of epidemic spreading in small-world networks: how prompt the recovery should be?, Int. J. Syst. Sci. 38 (5)(2007) 400-407.

[22] N. Madar, T. Kalisky, R. Cohen, D. ben-Avraham, and S. Havlin, Eur. Phys. J. B 38, (2004) 269.

[23] Xinchu Fu, Michael Small, David M. Walker, and Haifeng Zhang, Epidemic dynamics on scale-free networks with piecewise linear infectivity and immunization, PHYSICAL REVIEW E 77, (2008) 036113.

[24] D. S. Callaway, M. E. J. Newman, S. H. Strogatz, and D. J.Watts, Phys. Rev. Lett. 85, (2000) 5468.

[25] R. Cohen, K. Erez, D. ben-Avraham, and S. Havlin, Phys. Rev.Lett. 85, (2000) 4626.

Maoxing Liu was born in Shandong Province on June 28 1978. I had completed Master of Science degree under the supervision of Professor Yicang Zhou in the Department of Applied Mathematics of Xi'an Jiaotong University. In my thesis work and the publications, several discrete HIV/AIDS models were studied. After graduated from Xi'an Jiaotong University, I have become a teacher in North University of China. I have given some HIV/AIDS models and impulsive models in epidemiology and ecology with my partners. My three-year research experiences help me into a researcher in Mathematical Biology. In 2009, I have finished my Ph.D in Fudan University, majored in applied mathematics.

My main mathematical interests are stability and bifurcation theory of differential equations, nonlinear dynamical systems, stochastic differential equations and numerical analysis. My main scientific interests lie in applying mathematical methods to models that arise in biology, especially epidemiology and ecology. My professional goal is to continue to develop and apply mathematical tools to problems arising in biology with an emphasis on collaborative and interdisciplinary research.

My research activities cover a wide range of fields including stochastic differential equations, complex networks, spatial pattern formation, and so on. In other recent work, we developed Lassalle invariable principle for discrete stochastic differential equations driven by Brownian motion. This method is applicable to a wide class of discrete systems. 
Y unli. Zhang was born in a rural family of Handan City in Hebei Province on June 24 1984. She graduated from middle school in 2003, then entered Handan College to major in mathematics from 2003 to 2006, and went on to read mathematics in Tang Shan Teacher's College from 2006 to 2008. She graduated in 2008, and earned Bachelor of Science. Now she is a postgraduate two students in the North University of China, and studies in biomathematics and complex network.
She has some work experience. In summer vacation and weekends, she usually as Private Teacher for high school students and Promoters in shopping malls. Now her work is assistant in the North University of China. She has a keen interest in complex network and infections disease fields. 